\title{
Erratum
}

Daniele Di Mascio, Cihat Sen*, Gabriele Saccone, Alberto Galindo, Amos Grünebaum, Jun Yoshimatsu, Milan Stanojevic, Asım Kurjak, Frank Chervenak and et al. ${ }^{a}$

\section{Risk factors associated with adverse fetal outcomes in pregnancies affected by Coronavirus disease 2019 (COVID-19): a secondary analysis of the WAPM study on COVID-19}

https://doi.org/10.1515/jpm-2020-0539

Erratum to: Daniele Di Mascio, Cihat Sen*, Gabriele Saccone, Alberto Galindo, Amos Grünebaum, Jun Yoshimatsu, Milan Stanojevic, Asım Kurjak, Frank Chervenak, et al. ${ }^{\mathrm{a}}$ Risk factors associated with adverse fetal outcomes in pregnancies affected by Coronavirus disease 2019 (COVID-19): a secondary analysis of the
WAPM study on COVID-19. J. Perinat. Med. 2020; volume 48, Issue 9, pp. 950-958. https://doi.org/10.1515/ jpm-2020-0355.

Due to a technical error, the author list at the end of this article is unfortunately incorrect. Elif Gül Yapar Eyi is not a co-author, and therefore, his name and affiliation should not appear in the list. The correct author list and affiliations read as follows:

Daniele Di Mascio, Cihat Sen, Gabriele Saccone, Alberto Galindo, Amos Grünebaum, Jun Yoshimatsu, Milan Stanojevic, AsımKurjak, Frank Chervenak, María José Rodríguez Suárez, Zita Maria Gambacorti-Passerini, María de los Angeles Anaya Baz, Esther Vanessa Aguilar Galán, Yolanda Cuñarro López, Juan Antonio De León Luis, Ignacio Cueto Hernández, Ignacio Herraiz, Cecilia Villalain, Roberta Venturella, Giuseppe Rizzo, Ilenia Mappa, Giovanni Gerosolima, Lars Hellmeyer, Josefine Königbauer, Giada Ameli, Tiziana Frusca, Nicola Volpe, Giovanni Battista Luca Schera, Stefania Fieni, Eutalia Esposito, Giuliana Simonazzi, Gaetana Di Donna, Aly Youssef, Anna Nunzia Della Gatta, Mariano Catello Di Donna, Vito Chiantera, Natalina Buono, Giulio Sozzi, Pantaleo Greco, Danila Morano, Beatrice Bianchi, Maria Giulia Lombana Marino, Federica Laraud, Arianna Ramone, Angelo Cagnacci, Fabio Barra, Claudio Gustavino, Simone Ferrero, Fabio Ghezzi, Antonella Cromi, Antonio Simone Laganá, Valentina Laurita Longo, Francesca Stollagli, Angelo Sirico, Antonio Lanzone, Lorenza Driul, Fabiana Cecchini D, Serena Xodo, Brian Rodriguez, Felipe Mercado-Olivares, Deena Elkafrawi, Giovanni Sisti, Rosanna Esposito, Antonio Coviello, Marco Cerbone, Maddalena Morlando, Antonio Schiattarella, Nicola Colacurci, Pasquale De Franciscis, Ilaria Cataneo, Marinella Lenzi, Fabrizio Sandri, Riccardo Buscemi, Giorgia Gattei, Francesca della Sala, Eleonora Valori, Maria Cristina Rovellotti, Elisa Done, Gilles Faron, Leonardo Gucciardo, Valentina Esposito, Flaminia Vena, Antonella Giancotti, Roberto Brunelli, Ludovico Muzii, Luigi Nappi, Felice Sorrentino, Lorenzo Vasciaveo, Marco Liberati, Danilo Buca, Martina Leombroni, Francesca Di Sebastiano, Luciano Di

${ }^{\mathrm{a}}$ Full author list and affiliations given at the end of the article.

*Corresponding author: Cihat Sen, Perinatal Medicine Center, Memorial BAH Hospital and mPerinatal Medicine Foundation, Istanbul 34367, Turkey, E-mail: csen@perinatal.org.tr

Daniele Di Mascio, Department of Maternal and Child Health and Urological Sciences, Sapienza University of Rome, Rome, Italy Gabriele Saccone, Department of Neuroscience, Reproductive Sciences and Dentistry, School of Medicine, University of Naples Federico II, Naples, Italy

Alberto Galindo and Frank Chervenak, Department of Obstetrics and Gynaecology, Fetal Medicine Unit, Maternal and Child Health and
Development Network, University Hospital 12 de Octubre, Complutense University of Madrid, Madrid, Spain Amos Grünebaum, Department of Obstetrics and Gynaecology, Lenox Hill Hospital, Zucker School of Medicine at Hofstra/Northwell, Hempstead, NY, USA

Jun Yoshimatsu, Department of Perinatology and Gynaecology, National Cerebral and Cardiovascular Center, Osaka, Japan Milan Stanojevic and Asım Kurjak, Department of Obstetrics and Gynaecology, Medical School University of Zagreb, Sveti Duh University Hospital, Zagreb, Croatia 
Tizio, Diego Gazzolo, Massimo Franchi, Quintino Cesare lanniciello, Simone Garzon, Giuliano Petriglia, Leonardo Borrello, Albaro José Nieto-Calvache, Juan Manuel Burgos-Luna, Caroline Kadji, Andrew Carlin, Elisa Bevilacqua, Marina Moucho, Pedro Viana Pinto, Rita Figueiredo, José Morales Roselló, Gabriela Loscalzo, Alicia Martinez-Varea, Vincente Diago, Jesús S Jimenez Lopez, Alicia Yeliz Aykanat, Stefano Cosma, Andrea Carosso, Chiara Benedetto, Amanda Bermejo, Otto Henrique May Feuerschuette, Ozlem Uyaniklar, Sakine Rahimli Ocakouglu, Zeliha Atak, Reyhan Gündüz, Esra Tustas Haberal, Bernd Froessler, Anupam Parange, Peter Palm, Igor Samardjiski, Chiara Taccaliti, Erhan Okuyan, George Daskalakis, Renato Augusto Moreira de Sa, Alejandro Pittaro, Maria Luisa Gonzalez-Duran, Ana Concheiro Guisan, Şerife Özlem Genç, Blanka Zlatohlávková, Anna Luengo Piqueras, Dolores Esteban Oliva, Aylin Pelin Cil, Olus Api, Panos Antsaklis, Liana Ples, loannis Kyvernitakis, Holger Maul, Marcel Malan, Albert Lila, Roberta Granese, Alfredo Ercoli, Giuseppe Zoccali, Andrea Villasco, Nicoletta Biglia, Ciuhodaru Madalina, Elena Costa, Caroline Daelemans, Axelle Pintiaux, Elisa Cueto, Eran Hadar, Sarah Dollinger, Noa A. Brzezinski Sinai, Erasmo Huertas, Pedro Arango, Amadeo Sanchez, Javier Alfonso Schvartzman, Liviu Cojocaru, Sifa Turan, Ozhan Turan, Maria Carmela Di Dedda, Rebeca Garrote Molpeceres, Snezana Zdjelar, Tanja Premru-Srsen, Lilijana Kornhauser Cerar, Mirjam Druškovič, Valentina De Robertis, Vedran Stefanovic, Irmeli Nupponen, Kaisa Nelskylä, Zulfiya Khodjaeva, Ksenia A. Gorina, Gennady T. Sukhikh, Giuseppe Maria Maruotti, Silvia Visentin, Erich Cosmi, Jacopo Ferrari, Alessandra Gatti, Daniela Luvero, Roberto Angioli, Ludovica Puri, Marco Palumbo, Giusella D’Urso, Francesco Colaleo, Agnese Maria Chiara Rapisarda, Ilma Floriana Carbone, Antonio Mollo, Giovanni Nazzaro, Mariavittoria Locci, Maurizio Guida, Attilio Di Spiezio Sardo, Pierluigi Benedetti Panici, Vincenzo Berghella, Maria Elena Flacco, Lamberto Manzoli, Giuseppe Bifulco, Giovanni Scambia, Fulvio Zullo and Francesco D’Antonio

Flaminia Vena, Antonella Giancotti, Roberto Brunelli, Ludovico Muzii and Pierluigi Benedetti Panici, Department of Maternal and Child Health and Urological Sciences, Sapienza University of Rome, Rome, Italy

Rosanna Esposito, Antonio Coviello, Marco Cerbone, Giuseppe Maria Maruotti, Giovanni Nazzaro, Mariavittoria Locci, Maurizio Guida, Attilio Di Spiezio Sardo, Giuseppe Bifulco and Fulvio Zullo, Department of Neuroscience, Reproductive Sciences and Dentistry, School of Medicine, University of Naples Federico II, Naples, Italy

Ignacio Herraiz and Cecilia Villalain, Department of Obstetrics and Gynaecology, Fetal Medicine Unit, Maternal and Child Health and Development Network, University Hospital 12 de Octubre, Complutense University of Madrid, Madrid, Spain

María José Rodríguez Suárez, Hospital Universitario Central de Asturias, Asturias, Spain

Zita Maria Gambacorti-Passerini, Department of Obstetrics and Gynaecology, Ciudad Real University General Hospital, Ciudad Real, Spain

María de los Angeles Anaya Baz and Esther Vanessa Aguilar Galán, Department of Obstetrics and Gynaecology, Ciudad Real University General Hospital, Ciudad Real, Spain; University of Castilla-La Mancha, Ciudad Real, Spain

Yolanda Cuñarro López, Juan Antonio De León Luis and Ignacio Cueto Hernández, Department of Obstetrics and Gynaecology, Fetal Medicine Unit, Maternal and Child Health and Development
Network, Gregorio Marañón Hospital, Complutense University of Madrid, Madrid, Spain

Roberta Venturella, Department of Obstetrics and Gynaecology, School of Medicine, Magna Graecia University of Catanzaro, Catanzaro, Italy

Giuseppe Rizzo, University of Roma Tor Vergata, Division of Maternal Fetal Medicine, Ospedale Cristo Re Roma, Rome, Italy; Department of Obstetrics and Gynaecology, The First I.M. Sechenov Moscow State Medical University, Moscow, Russia

Ilenia Mappa, University of Roma Tor Vergata, Division of Maternal Fetal Medicine, Ospedale Cristo Re Roma, Rome, Italy

Giovanni Gerosolima, Department of Obstetrics and Gynaecology, Ospedale AOSG Moscati, Avellino, Italy

Lars Hellmeyer, Josefine Königbauer and Giada Ameli, Department of Gynaecology and Obstetrics, Vivantes Klinikum im Friedrichshain, Berlin, Germany

Tiziana Frusca, Nicola Volpe, Giovanni Battista Luca Schera and Stefania Fieni, Department of Obstetrics and Gynaecology, University of Parma, Parma, Italy

Eutalia Esposito, Department of Obstetrics and Gynaecology, Ospedale di San Leonardo, Castellammare di Stabia, Italy

Giuliana Simonazzi, Gaetana Di Donna, Aly Youssef and Anna Nunzia Della Gatta, Department of Obstetrics and Gynaecology, 
University of Bologna, Sant'Orsola- Malpighi University Hospital, Bologna, Italy

Mariano Catello Di Donna, Vito Chiantera, Natalina Buono and Giulio Sozzi, Department of Gynaecologic Oncology, University of Palermo, Palermo, Sicilia, Italy

Pantaleo Greco, Danila Morano, Beatrice Bianchi and Maria Giulia Lombana Marino, Department of Medical Sciences, Section of Obstetrics and Gynaecology, Azienda Ospedaliera-Universitaria Sant'Anna, University of Ferrara, Ferrara, Italy

Federica Laraud, Arianna Ramone, Angelo Cagnacci, Fabio Barra, Claudio Gustavino and Simone Ferrero, Academic Unit of Obstetrics and Gynaecology, IRCCS Ospedale Policlinico, San Martino, Genova, Italy

Fabio Ghezzi, Antonella Cromi and Antonio Simone Laganà, Department of Obstetrics and Gynaecology, "Filippo Del Ponte” Hospita University of Insubria, Varese, Italy

Valentina Laurita Longo, Department of Obstetrics and Gynaecology, Fondazione Policlinico Universitario A Gemelli IRCCS Università Cattolica del Sacro Cuore, Rome, Italy; Istituto di Clinica Ostetrica e Ginecologica, Università Cattolica del Sacro Cuore, Rome, Italy; and Queen Margaret University, Institute for Global Health and Development, Edinburgh, UK

Francesca Stollagli and Ludovica Puri, Istituto di Clinica Ostetrica e Ginecologica, Università Cattolica del Sacro Cuore, Rome, Italy

Angelo Sirico and Giovanni Scambia, Department of Obstetrics and Gynaecology, Fondazione Policlinico Universitario A Gemelli IRCCS - Università Cattolica del Sacro Cuore, Rome, Italy

Antonio Lanzone, Department of Obstetrics and Gynaecology, Fondazione Policlinico Universitario A Gemelli IRCCS - Università Cattolica del Sacro Cuore, Rome, Italy; Istituto di Clinica Ostetrica e Ginecologica, Università Cattolica del Sacro Cuore, Rome, Italy

Lorenza Driul, Fabiana Cecchini D and Serena Xodo, Clinic of Obstetrics and Gynaecology, University of Udine, Udine, Italy

Brian Rodriguez, Felipe Mercado-Olivares, Deena Elkafrawi and Giovanni Sisti, Department of Obstetrics and Gynaecology, New York Health and Hospitals/Lincoln Bronx, The Bronx, NY, USA

Maddalena Morlando, Antonio Schiattarella, Nicola Colacurci and Pasquale De Franciscis, Department of Woman, Child and General and Specialized Surgery, University of Campania Luigi Vanvitelli, Naples, Italy
Ilaria Cataneo, Marinella Lenzi and Fabrizio Sandri, Unit of Obstetrics and Gynaecology, Ospedale Maggiore, Bologna, Italy

Riccardo Buscemi, Giorgia Gattei, Francesca della Sala and Maria Cristina Rovellotti, Department of Translational Medicine, University of Eastern Piedmont, Novara, Italy

Eleonora Valori, Department of Translational Medicine, University of Eastern Piedmont, Novara, Italy; Hospital Castelli, Verbania, Italy

Elisa Done, Gilles Faron and Leonardo Gucciardo, UZ Brussel, Universitair Ziekenhuis, Brussel, Belgium Valentina Esposito, University of Milan, Milan, Italy

Luigi Nappi, Felice Sorrentino and Lorenzo Vasciaveo, Department of Obstetrics and Gynaecology, Department of Medical and Surgical Sciences, University of Foggia, Foggia, Italy

Marco Liberati, Danilo Buca, Martina Leombroni, Francesca Di Sebastiano, Luciano Di Tizio and Francesco D’Antonio, Centre for High Risk Pregnancy and Fetal Care, University of Chieti, Department of Obstetrics and Gynaecology, Chieti, Italy

Diego Gazzolo, Neonatal Intensive Care Unit, University of Chieti, Chieti Italy

Massimo Franchi, Quintino Cesare lanniciello and Simone Garzon, Department of Obstetrics and Gynaecology, AOUI Verona, University of Verona, Verona, Italy

Giuliano Petriglia and Leonardo Borrello, Maternal and Child Health Department, Santa Maria Hospital, Terni, Italy

Albaro Josè Nieto-Calvache and Juan Manuel Burgos-Luna, Fundación Valle del Lili, Tertiary Obstetric Unit, Cali, Colombia; Postgraduate Department, Universidad Icesi, Cali, Colombia

Caroline Kadji, Andrew Carlin and Elisa Bevilacqua, Department of Obstetrics and Gynaecology, University Hospital Brugmann, Université Libre de Bruxelles, Brussels, Belgium

Marina Moucho, Pedro Viana Pinto and Rita Figueiredo, Department of Obstetrics and Gynaecology, Centro Hospitalar e Universitário São João, Porto, Portugal

José Morales Roselló, Gabriela Loscalzo, Alicia Martinez- Varea and Vincente Diago, Servicio de Obstetricia y Ginecología, Hospital Universitario y Politécnico La Fe, Valencia, Spain

Jesús S. Jimenez Lopez, Hospital Regional Universitario de Málaga, Malaga, Spain 
Alicia Yeliz Aykanat, Department of Obstetrics and Gynaecology, Istanbul University-Cerrahpasa Medical School, Istanbul, Turkey

Stefano Cosma, Andrea Carosso and Chiara Benedetto, Department of Obstetrics and Gynaecology, Sant'Anna Hospital, University of Turin, Turin, Italy

Amanda Bermejo, Hospital Universitario de Móstoles, Mostoles, Spain

Otto Henrique May Feuerschuette, Departamento de Ginecologia e Obstetrícia, Hospital Universitário Polydoro Ernani, Santiago, Brazil

Ozlem Uyaniklar, Sakine Rahimli Ocakouglu and Zeliha Atak, Bursa City Hospital, Bursa, Turkey

Reyhan Gündüz, Department of Obstetrics and Gynaecology, University of Dicle, Diyarbakir, Turkey

Esra Tustas Haberal, Hisar Intercontinental Hospital, Istanbul, Turkey

Bernd Froessler, Anupam Parange and Peter Palm, Department of Anaesthesia, Lyell McEwin Hospital, Adelaide, Australia

Igor Samardjiski, University Clinic of Obstetrics and Gynaecology, Skopje, North Macedonia Chiara Taccaliti, Ospedale Generale Regionale “F. Miulli”, Acquaviva delle Fonti, Italy

Erhan Okuyan, Batman Maternity and Child Health Hospital, Batman, Turkey

George Daskalakis and Panos Antsaklis, Alexandra Hospital National and Kapodistrian, University of Athens, Athens, Greece

Renato Augusto Moreira de Sa, Assistência Obstétrica do Grupo Perinatal, Rio de Janeiro, Brazil

Alejandro Pittaro, Hospital Raul F. Larcade, Buenos Aires, Argentina

Maria Luisa Gonzalez-Duran and Ana Concheiro Guisan, Alvaro Cunqueiro University Hospital of Vigo, Vigo, Spain

Serife Özlem Genç, Karaman Public Hospital, Karaman, Turkey

Blanka Zlatohlávková, Department of Obstetrics and Gynaecology, Division of Neonatology, General Hospital in Prague and First Faculty of Medicine, Charles University, Prague, Czech Republic

Anna Luengo Piqueras and Dolores Esteban Oliva, Hospital Universitari Germans Trias i Pujol, Barcelona, Spain
Aylin Pelin Cil and Olus Api, American Hospital, Istanbul, Turkey

Liana Ples, Department of Obstetrics and Gynaecology, Saint John Hospital, UMF Carol Davila, Bucharest, Romania

Ioannis Kyvernitakis, Holger Maul and Marcel Malan, Asklepios Hospital Barmbek, Hamburg, Germany

Albert Lila, Regional Hospital Gjakova, Kosovo, Republic of Kosovo

Roberta Granese, Alfredo Ercoli and Giuseppe Zoccali, Department of Obstetrics and Gynaecology, University of Messina, Messina, Italy

Andrea Villasco and Nicoletta Biglia, Academic Division of Obstetrics and Gynaecolog, Mauriziano Umberto I Hospital, University of Turin, Turin, Italy

Ciuhodaru Madalina, Universitatea de Medicină și Farmacie Grigore T. Popa Iași, Iasi, Romania

Elena Costa, Caroline Daelemans and Axelle Pintiaux, Department of Obstetrics and Gynaecology, Hospital Erasme, Cliniques Universitaires de Bruxells, Brussels, Belgium

Elisa Cueto, Hospital Virgen De La Luz, Cuenca, Spain

Eran Hadar, Sarah Dollinger and Noa A. Brzezinski Sinai, Helen Schneider Hospital for Women, Rabin Medical Center, PetachTikva and Sackler Faculty of Medicine, Tel-Aviv University, TelAviv, Israel

Erasmo Huertas, Pedro Arango and Amadeo Sanchez, Instituto Nacional Materno Perinatal, Lima, Peru

Javier Alfonso Schvartzman, Centro de Educación Médica e Investigaciones Clínicas "Norberto Quirno", Buenos Aires, Argentina

Liviu Cojocaru, Sifa Turan and Ozhan Turan, Department of Obstetrics, Gynaecology and Reproductive Science, University of Maryland Medical Center, Baltimore, MD, USA

Maria Carmela Di Dedda, Department Gynaecology and Obstetrics, Fornaroli Hospital, Magenta, Italy

Rebeca Garrote Molpeceres, University Clinic Hospital of Valladolid, Valladolid, Spain

Snezana Zdjelar, Kbc Dr Dragisa Misovic Dedinje Belgrade, Beograd, Serbia 
Tanja Premru-Srsen, Lilijana Kornhauser Cerar and Mirjam Druškovic, Department of Perinatology, University Medical Center, Medical Faculty, University of Ljubljana, Ljubljana, Slovenia

Valentina De Robertis, Fetal Medicine Unit, Di Venere Hospital, Bari, Italy

Vedran Stefanovic, Irmeli Nupponen and Kaisa Nelskylä, Department of Obstetrics and Gynaecology, Neonatology and Intensive Care, Helsinki University Hospital and University of Helsinki, Helsinki, Finland

Zulfiya Khodjaeva, Ksenia A. Gorina and Gennady T. Sukhikh, National Medical Research Center for Obstetrics, Gynaecology and Perinatology, Moscow, Russia

Silvia Visentin, Erich Cosmi and Jacopo Ferrari, Department of Woman' and Child's Health, University of Padova, Padova, Italy
Alessandra Gatti, Daniela Luvero and Roberto Angioli, Campus Bio Medico, University of Rome, Rome, Italy

Marco Palumbo, Giusella D’Urso, Francesco Colaleo and Agnese Maria Chiara Rapisarda, Department of General Surgery and Medical Surgical Specialties, University of Catania, Catania, Italy

Ilma Floriana Carbone, Ospedale Maggiore Policlinico Milano, Milan, Italy

Antonio Mollo, University of Salerno, Salerno, Italy

Vincenzo Berghella, Maternal Fetal Medicine Division, Sidney Kimmel Medical College at Thomas Jefferson University, Obstetrics and Gynaecology Department, Philadelphia, PA, USA

Maria Elena Flacco and Lamberto Manzoli, Department of Medical Sciences, University of Ferrara, Ferrara, Italy 